\title{
Organización de la actividad preventiva y gestión de la seguridad y salud laboral en la minería española: experiencia de las empresas certificadas ISO 900 I
}

\author{
Organization of preventive activity and occupational health and safety \\ management in Spanish mining: experiencie of ISO 9001 certified firms
}

\author{
Carmen Escanciano García-Miranda, Beatriz Fernández Muñiz, Ana Suárez Sánchez, \\ Departamento de Administración de Empresas. Universidad de Oviedo.
}

cescan@uniovi.es beatrizf@uniovi.es suarezana@uniovi.es

Fecha de recepción: 07-08-09

Fecha de aceptación: 03-03-10

Resumen: La preocupación por la seguridad y la salud en el trabajo está presente en todos los sectores de actividad, pero especialmente en aquellos cuyas labores pueden suponer un mayor grado de riesgo: este es el caso del sector minero. La legislación española en materia preventiva obliga a las empresas a integrar la prevención de riesgos laborales en su sistema de gestión indicando la modalidad de organización de la actividad preventiva elegida. El presente trabajo evidencia que para las empresas mineras, el sistema de gestión de la seguridad y salud laboral OHSAS I 800 I constituye un marco adecuado para el cumplimiento de la legislación, al tiempo que asegura la protección de la salud y seguridad de los trabajadores.

Palabras clave: minería, seguridad, servicios de prevención, OHSAS I800।.

Abstract: Concern for safety and health at work is present in all sectors, but especially in those whose work may involve a higher degree of risk: this is the case of the mining sector.

Spanish legislation on safety obliges companies to integrate the prevention of occupational risks in their management system indicating the organization mode of the chosen preventive activity. This paper shows that for mining companies, the occupational health and safety management systems OHSAS I800I is an appropriate framework for compliance, as well as ensuring the protection of health and safety of workers.

Key words: mining, safety, prevention services, OHSAS I800I.

\section{Introducción}

Varios años después de la entrada en vigor del nuevo marco normativo en materia de prevención de riesgos laborales, la siniestralidad laboral continúa siendo uno de los problemas fundamentales de la sociedad y economía española, al alcanzarse la cifra de I.822. 146 accidentes de trabajo en el año 2007 (Ministerio de Trabajo e Inmigración, 2008). Las elevadas tasas de accidentalidad que prevalecen en la actualidad sitúan al país a la cabeza de la Unión Europea y provocan un significativo coste humano y una pérdida del potencial económico y de la productividad de las organizaciones y del país en general. En este sentido, el Ministerio de Trabajo y Asuntos Sociales estimó en el año 1996 una pérdida de productividad de la economía española, como consecuencia de los accidentes de trabajo, del 3\% del PIB nacional obtenido ese mismo año ( 13.280 millones de euros) y en el año 2002 se contabilizaron 2 1.597.604 de jorna- das de trabajo perdidas (Ministerio de Trabajo y Asuntos Sociales, 2002).

Si bien es un problema generalizado en todos los sectores es especialmente acusado en el sector de la minería, dada la peligrosidad intrínseca de la actividad. La minería se considera una industria especial con comunidades muy interrelacionadas y trabajadores que realizan un trabajo sucio y peligroso. Los mineros tienen que trabajar en un entorno laboral en constante transformación. Algunos trabajan sin luz natural o ventilación, excavando la tierra, extrayendo material y, al mismo tiempo, tomando medidas para evitar que se produzca una reacción inmediata en los estratos adyacentes (Organización Internacional del Trabajo, 2002). A pesar de los importantes esfuerzos realizados en muchos países, la tasa mundial de víctimas mortales, lesiones y enfermedades entre los trabajadores del sector muestra que, en la mayoría de ellos, la minería sigue siendo el 
trabajo más peligroso en relación con el número total de trabajadores dedicados a esta actividad (Jennings, 1998). Según el Ministerio de Trabajo e Inmigración, el índice de incidencia de la industria extractiva, que relaciona los accidentes en este sector con el número de personas que trabajan en el mismo, se situaba en el año 2007 en 44.04 I muy por encima de la media nacional, situada en 5.760,3.

La minería española, pese al fuerte proceso de reestructuración al que se ha visto sometida, factura actualmente unos 6.000 millones de euros y aporta el 4,4\% del Valor Añadido Bruto de la industria nacional. Según datos de la Subdirección General de Minas del Ministerio de Industria,Turismo y Comercio, el número de explotaciones mineras en el año 2004 ascendía a 6.000 y empleaban directamente a más de 80.000 personas, lo que supone un $2,2 \%$ de la población activa española en el sector industrial. Es preciso destacar la importancia de algunas extracciones mineras para las economías de las comarcas en determinadas regiones, como por ejemplo en el Principado de Asturias. En el caso particular de Asturias, la minería del carbón, empleando directamente a poco más del $1 \%$ de los trabajadores de la región, soporta más del I I\% de los accidentes laborales (Castaño, 2007).

Por todo ello, la actividad minera tiene importantes repercusiones económicas, ambientales, laborales y sociales, tanto en los países o regiones en que se practica como a escala global. El entorno en el que los trabajadores desarrollan su actividad y las condiciones en las que realizan su trabajo se configuran, pues, como elementos de vital importancia a la hora de velar por la salud y seguridad de los empleados. Así, las inversiones en seguridad y la formación constituyen los pilares fundamentales para la reducción de la siniestralidad laboral en la minería.

Con la globalización de la economía, la mayor preocupación por la seguridad a nivel mundial y el éxito de los sistemas de gestión de la calidad y de gestión medioambiental normalizados, las empresas comenzaron a demandar un modelo de gestión de la seguridad y salud laboral que siguiera los mismos principios de gestión que las normas en las que aquellos se basan, de modo que fuera fácilmente integrable con las mismas (Rubio, 200I). Con este propósito, surge OHSAS I 800 I, cuya última versión ha visto la luz en el año 2007. El estándar internacional OHSAS | 800 | es de carácter voluntario y establece requisitos para la mejora de las condiciones de trabajo, aunque muchos de ellos están implícitos en la propia le- gislación. Dadas sus múltiples ventajas, el estándar está teniendo una gran aceptación en organizaciones de todo tipo a nivel mundial. En el sector minero, la implantación del sistema de gestión de la seguridad y salud en el trabajo OHSAS I800 I parece traducirse en una reducción en el número de accidentes superior a la caída en el empleo, permitiendo la reducción efectiva de las tasas de siniestralidad. Así pues, este estándar se configura como un elemento fundamental para erradicar los accidentes laborales.

Bajo este contexto se plantea la realización de este trabajo, donde se ha tomado como referencia la industria minera española y, más concretamente, las empresas que poseen certificación ISO 9001:2000. La razón es que, tal y como apuntan diversos estudios, la mayoría de las empresas que han establecido un sistema de gestión medioambiental o un sistema de gestión de la seguridad y salud en el trabajo han comenzado por la certificación de sus sistemas de gestión de la calidad (Salomone, 2008; Zeng et al., 2008). Este trabajo pretende alcanzar un doble objetivo. En primer lugar, pretende analizar la modalidad más frecuente de organización de las actividades preventivas en la industria extractiva y, en segundo lugar, valorar la gestión de la seguridad y salud en el trabajo en este tipo de empresas y, más concretamente, analizar la experiencia de las empresas que han optado por la implantación del estándar OHSAS I800।.

\section{Organización de las actividades preventivas}

La Ley 31/1995, de Prevención de Riesgos Laborales, modificada por la Ley 54/2003, de reforma del marco normativo de la prevención de riesgos laborales, establece en el artículo 16 que la prevención de riesgos laborales deberá integrarse en el sistema general de gestión de la empresa, tanto en el conjunto de sus actividades como en todos los niveles jerárquicos de ésta, a través de la implantación y aplicación de un Plan de prevención de riesgos laborales. Este Plan incluirá: I) la identificación de la empresa, de su actividad productiva, el número y características de los centros de trabajo, el número de trabajadores y sus características con relevancia en la prevención de riesgos laborales; 2) la estructura organizativa de la empresa, identificando las funciones y responsabilidades que asume cada uno de sus niveles jerárquicos y los respectivos cauces de comunicación entre ellos, en relación con la prevención de riesgos laborales; 3 ) la organización de la producción en cuanto a la identi- 
ficación de los distintos procesos técnicos y las prácticas y los procedimientos organizativos existentes en la empresa, en relación con la prevención de riesgos laborales; 4) la organización de la prevención en la empresa, indicando la modalidad preventiva elegida y los órganos de representación existentes; y 5) la política, los objetivos y metas que en materia preventiva pretende alcanzar la empresa, así como los recursos humanos, técnicos, materiales y económicos de los que va a disponer al efecto. Los instrumentos esenciales para la gestión y aplicación del Plan de Prevención de Riesgos Laborales son la Evaluación de Riesgos y la Planificación de la Actividad Preventiva. Ambos instrumentos aparecen regulados en la Capítulo II del Real Decreto 39//997, por el que se aprueba el Reglamento de los Servicios de Prevención, modificado por el Real Decreto 604/2006 y el Real Decreto 337/2010.

Si los resultados de la Evaluación pusieran de manifiesto situaciones de riesgo, se determinarán las actividades preventivas necesarias para eliminar o reducir y controlar tales riesgos. Dichas actividades serán objeto de planificación, incluyendo para cada actividad preventiva las prioridades, el plazo para llevarla a cabo, la designación de responsables y los recursos humanos y materiales necesarios para su ejecución (arts. 8 y 9 del Real Decreto 39/1997). Posteriormente, se debe asegurar la efectiva ejecución de las actividades preventivas incluidas en la planificación, efectuando para ello un seguimiento continuo de la misma.

En el caso de las industrias extractivas la obligación de integración y planificación de la prevención se materializa en el Documento de Seguridad y Salud que explicita la manera en que la actividad preventiva se ha integrado en el sistema de gestión empresarial (Real Decreto 1389/1997, por el que se aprueban las disposiciones mínimas destinadas a proteger la seguridad y la salud de los trabajadores de las industrias extractivas). Un documento que ha de ser aprobado por la autoridad competente, en este caso, la autoridad minera, y que es requisito previo imprescindible para obtener la concesión o autorización administrativa para la explotación del yacimiento. Es decir, la prevención del riesgo laboral es un factor más a tener en cuenta en la gestión diaria de la empresa, cuya integración en el sistema general de gestión, así como la correcta planificación y organización de actividades, es una obligación y no una simple opción (artículo 2 de la Ley 54/2003).

La correcta elaboración, puesta en práctica y control del plan de prevención exige una previa organización de las actividades a realizar. La organización es un proceso de descripción y agrupación del trabajo, definición y delegación de funciones y autoridad y establecimiento de relaciones, con el propósito de que el personal trabaje unido lo más eficazmente posible (Niederleytner et al., 1996). Así pues, la organización preventiva podría definirse como el conjunto de relaciones y personas que, integradas en la estructura organizativa de la empresa, asegurarán el cumplimiento de los objetivos generales y políticas definidas por la dirección en la materia, así como la administración e implementación de todas las actividades relacionadas con la función preventiva.

La organización de la actividad preventiva consiste, por tanto, en establecer los recursos humanos y materiales necesarios, incluidos los financieros, con el fin de que los miembros de la organización puedan desarrollar correctamente sus funciones preventivas. La dirección debe elegir la estructura organizativa más idónea y dar a conocer el organigrama general de la empresa en el que se determinen las funciones a desarrollar por cada uno de sus miembros. Dentro de este organigrama deberán estar claramente establecidas las funciones y las responsabilidades en materia de prevención de todos los elementos de la empresa, así como la autoridad del órgano encargado de la función preventiva y sus relaciones con los restantes de la empresa.

La Ley de Prevención de Riesgos Laborales resta libertad al empresario para organizar la actividad preventiva, en cuanto a los medios materiales, personales, instalaciones o servicios. Dichas opciones están limitadas a beneficio de la operatividad y de la eficacia de las tareas preventivas (González et al., 1997; González, 1999). De este modo, el empresario, para cumplir con la obligación de protección de los trabajadores, deberá asignar los recursos necesarios para el desarrollo de las actividades preventivas. Estos recursos serán organizados en función de alguna de las siguientes modalidades:

- Asumiendo personalmente tales actividades.

- Designando a uno o varios trabajadores para llevarla a cabo.

- Constituyendo un Servicio de Prevención Propio.

- Concertando un Servicio de Prevención Ajeno.

El propio empresario podrá encargarse de la prevención en la empresa, exclusivamente cuando la empresa tenga hasta diez trabajadores, la actividad de 
la misma no esté incluida en el Anexo I del Real Decreto 39/1997, es decir, no sea considerada como peligrosa, desarrolle su actividad profesional de forma habitual en el centro de trabajo y tenga las capacidades correspondientes a las actividades preventivas que va a desarrollar (Art. I I del Real Decreto 39/I997, modificado por el Real Decreto 337/20 I0). Si se incumple alguno de estos requisitos, como es el caso del sector de la minería, que figura en el citado Anexo I, el empresario deberá designar trabajadores o recurrir a servicios de prevención si la designación de trabajadores resulta insuficiente. Los trabajadores designados deberán tener la capacidad correspondiente a las funciones a desempeñar y deberán ser los necesarios para desarrollar adecuadamente sus funciones.

El Servicio de Prevención viene definido por el artículo 31.2 de la Ley 31/1995 como el «conjunto de medios humanos y materiales necesarios para realizar las actividades preventivas a fin de garantizar la adecuada protección de la seguridad y salud de los trabajadores, asesorando y asistiendo para ello al empresario, a los trabajadores y a sus representantes y a los órganos de representación especializados». Estos servicios se encargarán de evaluar los riesgos de la empresa, diseñar el plan de prevención, determinar prioridades en la adopción de medidas preventivas, formar e informar a los trabajadores, realizarán la prestación de primeros auxilios y el diseño del plan de emergencia y serán los encargados de realizar la vigilancia de la salud de los trabajadores.

El empresario tendrá la obligación de constituir un Servicio de Prevención Propio, definido en el artículo I 0.2 del Real Decreto 39/1997 como «el conjunto de medios materiales y humanos de la empresa necesarios para la realización de las actividades de prevención», cuando la empresa tenga más de 500 trabajadores o más de 250 si la actividad de la misma está considerada como peligrosa (caso de la minería). Asimismo, será obligatoria su constitución cuando, no alcanzando el número de trabajadores anterior, lo decida la Autoridad Laboral previo informe de la Inspección de Trabajo y Seguridad Social (artículo I 4 del Real Decreto 39/1997). En cualquier otro caso podrá concertar las actividades preventivas con una entidad especializada ajena a la empresa, es decir, podrá recurrir a un Servicio de Prevención Ajeno, definido por el citado Real Decreto como «el prestado por una entidad especializada que concierte con la empresa la realización de actividades de prevención, el asesoramiento y el apoyo que precise en función de los tipos de riesgos» (artículo 10.2). Asimismo, el artículo 21 del Real Decreto 39/1997 es- tablece la posibilidad de constituir servicios de prevención mancomunados (servicios propios compartidos por varias entidades) entre aquellas empresas que desarrollen simultáneamente actividades en un mismo centro de trabajo, edificio o centro comercial, siempre que quede garantizada su operatividad y eficacia.

No existe un único sistema válido de organización de la prevención, ya que dependerá de cómo esté organizada la empresa y la cultura que en ella exista. El modelo más eficaz en cada caso es aquel que se integre plenamente a la propia organización productiva, logrando que directivos, técnicos, mandos y trabajadores asuman las responsabilidades que tienen en la materia. Con independencia de la modalidad de organización preventiva elegida es necesario disponer siempre en todo centro de trabajo de personas involucradas en tareas de coordinación, seguimiento y control de la gestión preventiva del riesgo laboral. Aunque es permisible legalmente que una empresa pueda concertar toda la actividad preventiva con un servicio de prevención ajeno, es muy aconsejable la existencia de un coordinador de prevención, es decir, que alguien del interior de la misma, y contando con el apoyo de la dirección, actúe de vínculo o de interlocutor y preste el apoyo logístico necesario para el correcto desarrollo e implantación del sistema (Bestratén y Marrón, 200 I). No se trata de desarrollar una estructura paralela o superpuesta a las ya existentes sin ninguna o pocas conexiones entre ellas, sino que se trata de adaptar la organización preventiva a la organización global de la empresa, aprovechando incluso, si las hubiere, estructuras organizativas desarrolladas para campos de acción afines, como calidad o medioambiente.

No obstante, las formas más frecuentes de organización son la organización en línea, la organización staff y la organización en grupos de trabajo (Cortés, 2000). En la organización en línea todas las funciones de la acción preventiva, gestión, ejecución y responsabilidades las realizan cada uno de los mandos de la empresa como una función más integrada en las actividades propias de su área de trabajo. En cambio, en la organización staff las funciones de la acción preventiva son realizadas por personal especializado en prevención de riesgos que actúan como asesores de los diferentes estamentos de la empresa. Parece ser que es la forma de organización más aconsejable siempre que dependan directamente de la Dirección de la empresa (Cortés, 2000). Por su parte, en la organización en grupos de trabajo la acción preventiva es realizada por grupos de trabajo (trabajadores y técnicos) similares a los círculos de calidad. 


\section{Los sistemas de gestión de la seguridad y salud en el trabajo en la industria minera}

La preocupación por la seguridad y la salud en el trabajo está presente en todos los sectores de actividad, pero especialmente en aquellos cuyas labores pueden suponer un mayor grado de riesgo. Este es el caso del sector minero, caracterizado por su peligrosidad y tradicionalmente vinculado a elevados índices de siniestralidad, asociados, en muchos casos, a la pérdida de vidas humanas. En España, la protección de la seguridad y la salud de los trabajadores en general, y la de los trabajadores del sector minero en particular, siempre ha sido muy importante. Una buena prueba de ello son las numerosas disposiciones legales y reglamentarias ' que tienen por objeto garantizar un nivel de protección adecuado frente a los riesgos derivados de las condiciones de trabajo. Estas disposiciones exigen, fundamentalmente, organización, planificación y control. En este sentido, los sistemas de gestión de la seguridad y salud en el trabajo se presentan como una herramienta ideal para la implantación de las actividades preventivas en las organizaciones, dotando a las mismas de unos medios para la gestión de la seguridad y salud laboral de una forma estructurada y planificada (Riesgo et al., 2005). Esta gestión sistemática de la seguridad y salud no sólo dará como resultado una reducción de los ratios de siniestralidad sino que, normalmente, generará un aumento de la productividad y de los resultados económicos y financieros de la empresa (O'Toole, 2002).

Existen en la actualidad diversos referentes y normas de carácter voluntario para el establecimiento de este tipo de sistemas ${ }^{2}$, siendo el modelo más aceptado y extendido en el mercado el definido por el estándar OHSAS I 800 I. En gran medida el secreto de su éxito reside en que ofrece la posibilidad de certificación, por lo que constituye una forma fácil de demostrar a los diferentes grupos de presión que la organización dispone de un buen sistema de gestión de la seguridad y salud en el trabajo. Además, desde su origen, ha sido diseñada para ser compatible con los estándares ISO en materia de calidad y me- dioambiente (Zeng et al., 2007). Como sostienen Duijm et al. (2008), los principios de la seguridad y salud laboral están íntimamente relacionados con los principios de gestión de la calidad en los que se fundamenta ISO $900 \mathrm{I}$, y la propia estructura de la norma está basada en el ciclo PDCA (Plan-Do-CheckAct) y el concepto de mejora continua. En el Anexo se pueden observar las analogías entre OHSAS I800 I, ISO 900 | e ISO | 400 I.

El objetivo fundamental del estándar OHSAS I 800 | es apoyar y promover buenas prácticas en materia de seguridad y salud en el trabajo a través de una gestión sistemática y estructurada. El estándar especifica los requisitos para la implantación de un sistema de gestión de la seguridad y salud en el trabajo que permita a una organización desarrollar e implementar una política de seguridad, establecer objetivos y procesos para alcanzar los compromisos de la política, tomar las acciones necesarias para mejorar su desempeño y demostrar la conformidad del sistema con los requisitos de dicho estándar, teniendo en cuenta las exigencias legales (AENOR, 2009). No obstante, el éxito del sistema depende del compromiso de todos los niveles y funciones de la organización y, especialmente, de la alta dirección (Fernández et al., 2009).

El estándar OHSAS, en su requisito 4.3.2., obliga a la empresa al conocimiento y compromiso de cumplimento de la legislación aplicable, lo que supone que la implantación de un sistema de gestión de la seguridad y salud en el trabajo basado en este estándar no sólo permite a cualquier organización fomentar un entorno de trabajo seguro, sino también cumplir con las obligaciones legales en un marco de mejora continua.

Sin embargo, a pesar de la exigente legislación española en materia de seguridad y salud laboral y del alto grado de cumplimiento de la misma por parte de las empresas (Sesé, 2002), la adopción de Sistemas de gestión de la seguridad y salud en el trabajo basados en OHSAS I800I sólo se presenta en aquéllas organizaciones que poseen experiencia previa en la implantación de otros sistemas de gestión, gene-

\footnotetext{
I Riesgo y penosidad están íntimamente asociados a la profesión minera, ya que son muchos los factores de riesgo ligados a esta actividad por su naturaleza, métodos de trabajo y lugar su desarrollo. Debido a estas características, la minería siempre ha sido objeto de una legislación especial. Entre ella destacan el Estatuto del Minero de 1983, el Reglamento General de Normas Básicas sobre Seguridad Minera de 1985, y el Real Decreto 1389/1997 por el que se aprueban las disposiciones mínimas destinadas a proteger la seguridad y la salud de los trabajadores de las industrias extractivas.

2 Las alternativas con las que cuenta una empresa en cuanto a modelos de gestión de la seguridad y la salud en el trabajo a implantar son múltiples, dada la ausencia de una norma internacional. Entre ellos destacan: las directrices Organización Internacional del Trabajo, la norma británica BS 8800 u OHSAS I8001.
} 
ralmente basados en la norma ISO 900 I (Riesgo et al., 2005; Fernández et al., 2009). Por ello, el presente estudio se centra en empresas del sector minero que poseen certificado de calidad ISO 900 I.

\section{Metodología}

Con la finalidad de conocer cómo las empresas mineras certificadas ISO 900 I organizan la actividad preventiva y gestionan la seguridad y salud laboral se ha llevado a cabo un estudio empírico de carácter exploratorio. Para ello, se ha considerado como población objetivo el conjunto de empresas ubicadas en España, tanto nacionales como extranjeras, integrantes del sector minero que a 31 de enero de 2007 contaban con un sistema de calidad certificado. Para determinar el tamaño de la población se contactó con las entidades acreditadas por ENAC (Entidad Nacional de Acreditación) para la certificación de sistemas de gestión de la calidad en el sector minero. De esta manera, se detectaron 222 empresas, de las que 203 pudieron ser localizadas. En mayo de 2007 se les remitió un cuestionario postal, diseñado tras una pormenorizada revisión de la literatura.

El cuestionario recogía información sobre la modalidad organizativa de cada disciplina preventiva, la existencia de algún sistema de gestión de la prevención de riesgos laborales (SPRL), así como los motivos para su implantación y beneficios derivados del mismo. Los motivos y beneficios del SPRL fueron medidos a través de varios ítems planteados en escalas Likert I-5, donde I = «totalmente en desacuerdo» y $5=$ «totalmente de acuerdo». Se estableció el 30 de septiembre como fecha límite para la recepción de las encuestas y el tratamiento de la información. Durante el mismo mes se realizó una revisión pormenorizada de las encuestas recibidas, que ocasionó el rechazo de varias de ellas por aportar información inconsistente internamente. Finalmente, tras la inspección y depuración de los cuestionarios cumplimentados, la información obtenida se sometió a tabulación y se efectuó un análisis exploratorio de los datos.

La muestra objeto de análisis quedó finalmente configurada por 104 empresas certificadas ISO 9000 del sector de la minería (CNAE I0) que operan en territorio nacional y que se corresponden con idéntico número de encuestas válidas. Las compañías que integran la muestra se hallan distribuidas en 15 de las 17 Comunidades Autónomas españolas, si bien Castilla-León y Galicia constituyen su ubicación más fre- cuente. Como se puede observar en la Figura I, se trata mayoritariamente de Pymes, de capital nacional, de las que el 44,5\% afirma pertenecer a un grupo empresarial. Con respecto a su facturación, ésta supera los 3 millones de euros en el 60\% de los casos. En cuanto a los mercados en los que operan, la mayor parte concentra su actividad en el mercado nacional, si bien el $31 \%$ afirma exportar sus productos a los países de su entorno más próximo, los del continente europeo.

Figura |

Tamaño de las empresas de la muestra (número de trabajadores)

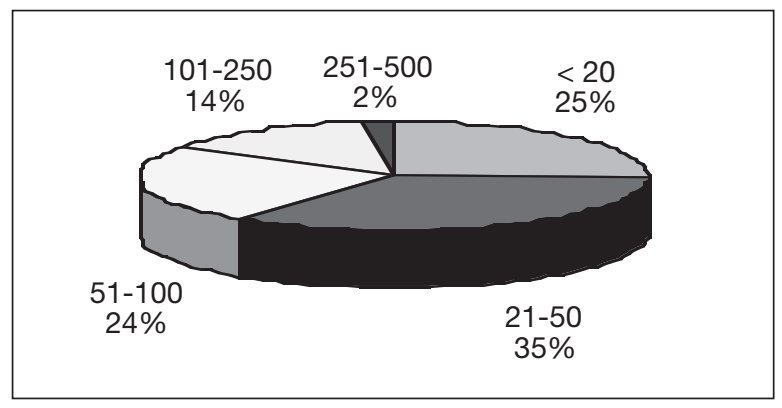

El 73\% de las integrantes de la muestra cuenta con un Departamento de Calidad con una antigüedad superior a los cuatro años, situándose la edad media de este tipo de departamentos en torno a los doce años. Esto pone de manifiesto que la preocupación por la calidad y su mejora en la empresa minera española no es tan reciente como cabría pensar. Al fin y al cabo el producto que ofrece se extrae directamente del medio natural para, en muchas ocasiones, ser transformado o bien incorporado junto con otros elementos a un proceso industrial por parte del cliente.

\section{Resultados}

LaTabla I recoge la modalidad de organización preventiva de cada una de las disciplinas que integra la prevención de riesgos laborales. En la misma se puede observar el predominio de los servicios de prevención ajenos en las cuatro grandes áreas de Seguridad en el Trabajo, Higiene Industrial, Ergonomía y Psicosociología Aplicada y Medicina del Trabajo. En la especialidad de Seguridad, el 92,86\% de las explotaciones mineras afirma tener concertado un servicio de prevención ajeno, el 5,1\% de las empresas dispone de un servicio de prevención propio, aunque el 2,04\% de éstas afirma compartirlo con otras entidades (servicio de prevención mancomunado). 
Sólo el 2,04\% de los centros cuenta con trabajadores designados.

En el área de Higiene Industrial la distribución es muy similar. El 91,68\% de las empresas ha contratado esta actividad con una entidad externa, el 5,21\% ha creado un servicio de prevención propio, el 2,08\% utiliza un servicio mancomunado y únicamente el I,04\% de las entidades ha designado trabajadores. Lo mismo ocurre en la especialidad de Ergonomía y Psicosociología Aplicada, donde el 91,67\% de las instituciones han optado por un servicio de prevención ajeno, el 5,21\% hace uso de un servicio de prevención mancomunado, el 2,08\% dispone de trabajadores designados y sólo el I,04\% dispone de un servicio de prevención propio.

Finalmente, en cuanto a la vigilancia de la salud o la medicina del trabajo las empresas de la muestra afirman que es llevada a cabo por un servicio de prevención ajeno en el 85,30\% de las mismas. Sin embargo, a diferencia del resto de especialidades, existen trabajadores designados en el | I,76\% de los centros. En cambio, solamente se han constituido servicios de prevención propios en el 1,96\% de las empresas y sólo un $0,98 \%$ hace uso de un servicio de prevención mancomunado.

Así pues, en el sector minero las actividades preventivas son realizadas fundamentalmente por servicios de prevención ajenos, pero no se trata de una característica exclusiva de este sector. El concierto de las actividades preventivas con un servicio externo está muy extendido en todo tipo de sectores (Fernández et al., 2005). Aunque estos servicios son una garantía para la efectiva prevención de los riesgos derivados del trabajo, puesto que están constituidos por un conjunto de recursos humanos altamente cualificados e independientes, el recurso a los mismos suele ser in- dicativo de la escasa integración de la actividad preventiva en la gestión general de la empresa.

Por otro lado, en el sector de la minería se puede afirmar que efectivamente existe relación entre el sistema de gestión de la calidad y el sistema de gestión de la prevención de riesgos laborales (SPRL), puesto que el $87 \%$ de las empresas integrantes de la muestra afirman contar con un SPRL ya implantado o en fase de implantación que tiene como referencia OHSAS I800। (Figura 2).

Figura 2

La empresa minera y el SGPRL

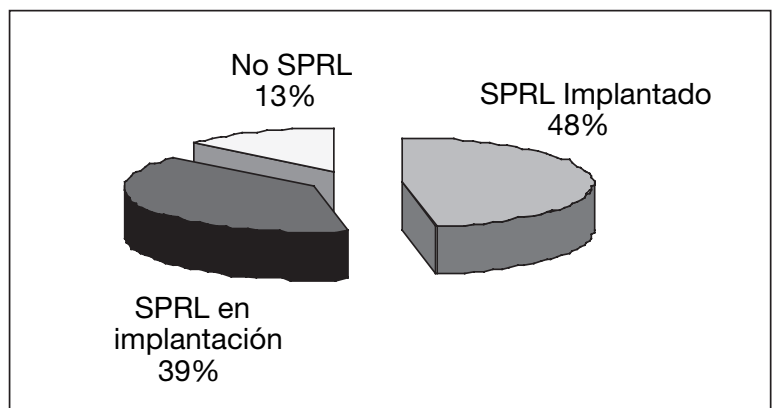

El aumento de interés en la adopción de estándares en el campo de la seguridad y salud laboral se ha visto acompañado por una demanda creciente de la certificación de estos sistemas (Cottam, 2000). La norma OHSAS I800I es certificable, sin embargo, podría considerarse que la exigencia de una certificación en el campo de la prevención de riesgos laborales no ha llegado, al menos de momento, a consolidarse. Probablemente la «juventud» de esta norma -su primera versión data de 1999-,y el hecho de no haber sido elaborada por ISO pudieran justificar este hecho. Sea como fuere, lo que sí que es

Tabla I

Modalidad de organización de la actividad preventiva

\begin{tabular}{|l|c|c|c|c|}
\hline \multirow{2}{*}{ MODALIDAD ADOPTADA } & \multicolumn{3}{|c|}{ ESPECIALIDAD PREVENTIVA } \\
\cline { 2 - 5 } & $\begin{array}{c}\text { Seguridad en } \\
\text { el trabajo (\%) }\end{array}$ & $\begin{array}{c}\text { Higiene } \\
\text { industrial (\%) }\end{array}$ & $\begin{array}{c}\text { Ergonomía psicosociología } \\
\text { aplicada (\%) }\end{array}$ & $\begin{array}{c}\text { Medicina } \\
\text { del trabajo (\%) }\end{array}$ \\
\hline Trabajador designado & 2,04 & 1,04 & 2,08 & 11,76 \\
\hline Servicio de prevención propio & 3,06 & 5,21 & 1,04 & 1,96 \\
\hline Servicio de prevención mancomunado & 2,04 & 2,08 & 5,21 & 0,98 \\
\hline Concierto con un servicio de prevención ajeno & 92,86 & 91,67 & 91,67 & 85,30 \\
\hline
\end{tabular}


cierto es que frente a las más de 800.000 certificaciones ISO 9001 y los cerca de 129.000 certificados ISO | 400 I, tan solo 15.000 organizaciones repartidas en 100 países cuentan con una certificación $\mathrm{OH}$ SAS I800I (Quejido, 2008). En el caso de las empresas de la muestra, tan solo un $5 \%$ de ellas en el momento de realización de este estudio poseían este certificado, lo que evidencia que este reconocimiento no se halla asentado en el sector minero.

Como se puede apreciar en la Tabla 2, los motivos de mayor peso en la decisión de establecer un SPRL en la empresa han sido conseguir una reducción del nivel de accidentalidad y lograr la conformidad con la legislación, lo que parece reflejar que la empresa ve en la normativa OHSAS I 800 I el modelo idóneo para lograr los fines previstos por la legislación en materia preventiva.

A ellos ha de unirse el interés en evitar posibles multas y sanciones -seguramente debidas a incumpli- mientos de las prescripciones legales-, satisfacer las exigencias de la Administración -entendidas, en el caso de la empresa minera, como el deber de actualizar anualmente el Documento de Seguridad y Salud (Fortuna y Comajuncosa, 2005)-, y mejorar la imagen de la empresa. Por otra parte, el deseo de complementar otros sistemas de gestión es otra motivación relevante y pone de manifiesto la existencia de similitudes entre aquéllos y el que nos ocupa.También, los trabajadores parecen haber jugado un papel importante a la hora de de implantar este SPRL, lo que, tal y como señala Petersen (2000), es un indicador de la existencia de una cultura de seguridad en la empresa.

Petersen (2000) también habla de la existencia de diversos beneficios asociados a la posesión de un SPRL. Como se puede apreciar en la Tabla 3, las integrantes de la muestra manifiestan haber obtenido la práctica totalidad de los beneficios propuestos con una intensidad superior al valor medio de la escala.

Tabla 2

Razones para la implantación de un SPRL

\begin{tabular}{|l|c|c|}
\hline \multicolumn{1}{|c|}{ RAZONES PARA LA IMPLANTACIÓN } & Media & Desv. $\boldsymbol{t}$ \\
\hline Reducir la accidentalidad & 4,73 & 0,77 \\
Conformidad con la legislación & 4,60 & 0,60 \\
Evitar multas y sanciones & 4,16 & 1,16 \\
Exigencia de la Administración & 3,72 & 1,34 \\
Mejora de la imagen de la empresa de cara al exterior & 3,62 & 1,18 \\
Complementar otros sistemas de gestión & 3,43 & 1,34 \\
Exigencia de los trabajadores & 3,28 & 1,13 \\
Mis competidores ya lo tienen & 2,08 & 1,22 \\
\hline
\end{tabular}

Tabla 3

Beneficios de un SPRL

\begin{tabular}{|l|c|c|}
\hline \multicolumn{1}{|c|}{ BENEFICIOS DE LA GESTIÓN PREVENTIVA } & Media & Desv. Típ. \\
\hline Cumplimiento de legislación sobre seguridad y salud laboral & 4,51 & 0,63 \\
Mejora del nivel de formación de los trabajadores & 4,09 & 0,76 \\
Mayor concienciación social & 4,06 & 0,67 \\
Mejora de la imagen de la empresa & 3,92 & 0,92 \\
Reducción de la accidentalidad & 3,83 & 1,06 \\
Incremento de la satisfacción de los trabajadores & 3,84 & 0,79 \\
Mejora del control y la gestión global de la empresa & 3,74 & 0,90 \\
Mejora de la eficiencia (reducción tiempos perdidos...) & 3,34 & 1,01 \\
Complementar otros sistemas de gestión & 3,37 & 1,27 \\
Ahorro de costes (seguros baratos, producción...) & 2,90 & 1,19 \\
\hline
\end{tabular}


En coherencia con los resultados obtenidos por Fernández et al. (2009), el beneficio más valorado ha sido el cumplimiento de la legislación, lo que en cierto modo es una consecuencia directa del compromiso adquirido por la empresa al elegir la norma OHSAS | 800 | a la hora de diseñar e implantar su sistema de gestión preventiva. Se trata, en cualquier caso, de beneficios interrelacionados entre sí, pues casi todos ellos son al mismo tiempo causa y efecto de los demás. Así, el segundo beneficio en importancia, la mejora del nivel de formación de los trabajadores en temas relacionados con la seguridad, junto con el citado en primer lugar, puede asociarse a la reducción de la tasa de accidentalidad. Lógicamente, todo ello refuerza la motivación de los trabajadores, que se muestran más satisfechos al desarrollar su labor en un ambiente de trabajo seguro, lo que confirma lo apuntado por Oliveira y Coelho (2002): «la prevención es el área en la que se pueden lograr los mayores niveles de satisfacción de los trabajadores». Por otra parte, la disminución de incidentes y accidentes laborales conlleva una reducción de interrupciones del proceso productivo, lo que es un efecto y a la vez redunda en un control y gestión de la empresa más eficiente, siendo capaz de prestar un servicio fiable sin excesivos tiempos de inactividad originados por percances relacionados con el trabajo. Todo ello implica una reducción de los costes de no calidad por fallos internos, con la correspondiente mejora en el sistema de gestión de la calidad de la empresa. Por consiguiente, se produce una mejora de la imagen de la empresa, que, previsiblemente, se vería potenciada en el caso de que ésta optara por la certificación de su SPRL.

Salomone (2008), en su estudio sobre los sistemas integrados de gestión en Italia, señala que uno de los inconvenientes aducidos por las empresas a la hora de implantar un SPRL es su alto coste. En este sentido, se preguntó a las empresas de la muestra si el coste y esfuerzo de la implantación de su sistema se había visto compensado o superado por los beneficios obtenidos, observando que el $64 \%$ de las empresas manifestaron que estos últimos habían superado con creces ${ }^{3}$ a los costes (Figura 3 ).

En definitiva, parece detectarse un balance global positivo en lo que a la implantación de un sistema de prevención de riesgos laborales basado en OHSAS | 800 | respecta, pero no tanto desde la perspectiva de su certificación.
Figura 3

Los beneficios de la implantación de un SPRL

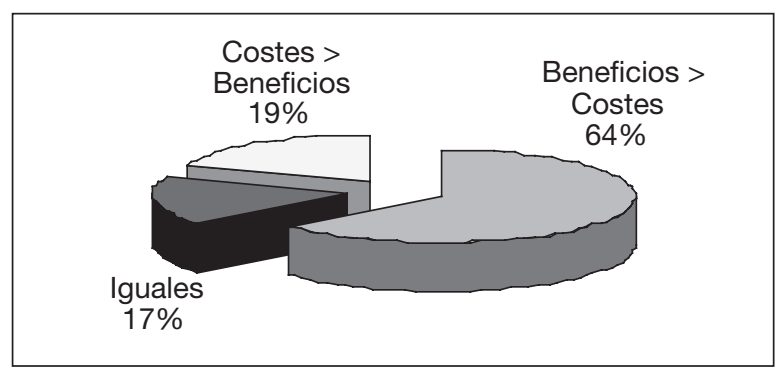

\section{Conclusiones}

La industria extractiva es considerada un sector de alto riesgo capaz de generar numerosos daños tanto personales como materiales. Por ello las entidades vinculadas a este sector deben realizar un elevado esfuerzo e implantar un adecuado sistema de gestión de la seguridad y salud laboral para prevenir todo tipo de accidentes e incidentes. En este trabajo se ha analizado tanto la gestión como la organización de las actividades preventivas en las entidades mineras certificadas ISO 900 I , alcanzando las siguientes conclusiones.

El análisis de la modalidad de organización de las actividades preventivas revela el gran uso que este tipo de empresas hace de los servicios de prevención ajenos, ya que las cuatro grandes áreas que configuran la prevención de riesgos laborales (seguridad, higiene, ergonomía y psicosociología y medicina) son concertadas con un servicio externo en más del $85 \%$ de los casos. Se detecta, pues, la escasez de estructuras internas, como los servicios de prevención propios o los trabajadores designados, lo que genera ciertas dudas sobre la efectiva integración de la prevención de riesgos laborales en la gestión general de la empresa, obligación del empresario recogida en el artículo 2 de la Ley 54/2003, de reforma del marco normativo de la prevención de riesgos laborales.

Por otro lado, la similitud y compatibilidad entre ISO 9001 y OHSAS 1800 I favorece la implantación de un sistema de gestión de la seguridad y salud en el trabajo en la industria minera. Este referente tiene una estructura semejante y comparte principios de gestión con los estándares de calidad y medio ambiente elaborados por la Organización Internacional

\footnotetext{
3 En previsión del reducido número de empresas que en general, y en el sector de la minería en particular, cuentan a día de hoy con un certificado OHSAS I800I, se decidió que se valorasen tan solo los efectos de la implantación de un SPRL y no su certificación, a fin de conseguir un número de respuestas que permitiera obtener información relevante.
} 
de Normalización. Sin embargo, hay que destacar el papel coadyuvante jugado por la rigurosa legislación de prevención de riesgos laborales que las empresas del sector han de cumplir para poder desarrollar su actividad. De hecho, lograr la conformidad con la legislación y su cumplimiento no sólo es la motivación fundamental para la implantación de los sistemas de prevención de riesgos laborales, sino también el beneficio más importante derivado de su establecimiento. De esta manera, OHSAS I800I no sólo contribuye a fomentar un entorno de trabajo seguro, sino a cumplir con las obligaciones legales en un marco de mejora continua, confirmando de esta manera lo apuntado por Zeng et al. (2007): «un sistema legal fuerte es un prerrequisito para una efectiva gestión de la seguridad».

Por lo que respecta a la certificación de estos sistemas, se observa que, a diferencia de lo que sucede en el ámbito de la calidad, no todas las empresas se muestran dispuestas a dar ese paso, tal vez por estimar suficientes los beneficios que proporciona la simple implantación del sistema o no considerarlo necesario. Apenas el 5\% de las empresas de la muestra afirman contar con la certificación OHSAS I800I. Tal vez la falta de demanda de la misma por parte de clientes y Administraciones Públicas podría justificar esta situación.

\section{Bibliografía}

AENOR (2009). OHSAS I 800 1:2007 Sistemas de gestión de la seguridad y salud en el trabajo. OHSAS 18002:2008 Sistemas de gestión de la seguridad y salud en el trabajoDirectrices para la implementación de OHSAS 18001:2007. AENOR: Madrid.

BESTRATEN, M. y MARRON, M.A. (200I). «Sistema de Gestión Preventiva: Organización y Definición de Funciones Preventivas», Nota Técnica de Prevención 565. Instituto Nacional de Seguridad e Higiene en el Trabajo.

CASTANO, P. (2007). «La minería aún es el segundo sector en Asturias con más accidentes después de la construcción», La Nueva España, 27 de septiembre.

CORTES, J.M. (2000). Técnicas de Prevención de Riesgos Laborales. Seguridad e Higiene del Trabajo, Editorial Tébar, Madrid.

COTTAM, M.P. (2000). «Certification of occupational health and safety management systems», Proceedings of the Conference on Foresight and Precaution, Edinburgh, vols. I-2, pp. 455-461.

DUIJM, N.J.; FIEVEZ, C.; GERBEC, M.; HAUPTMANNS, U. y Konstandinidou, M. (2008). «Management of health, safety and environment in process industry», Safety Science, dii: 10 I 0 I 6/j.ssci.2007.I I.03.

FERNANDEZ, B., MONTES, J.M. y VAZQUEZ, C.J. (2005). Cómo crear un entorno de trabajo seguro. Organización y gestión económica de la salud laboral, EditorialThomsonParaninfo, Madrid.

FERNANDEZ, B., MONTES, J.M., VAZQUEZ, C.J., SANCHEZ-TOLEDO, A. y FERNANDEZ, F. (2009). «Certificación OHSAS I800 I: la opinión de las empresas auditadas», Gestión Práctica de Riesgos Laborales 60 , pp. |2-2|.

FORTUNA, J. y COMAJUNCOSA, J. (2005). «El documento sobre seguridad y salud: un sistema de gestión de la prevención para las canteras»), Actas del IX Congreso de Ingeniería de Organización, Gijón.

GONZALEZ, S. (1999). «La Organización de la Prevención por el Empresario: los Servicios de Prevención», Temas Laborales 50, pp. 19-47.

GONZALEZ, S., MORENO, A. y FERNANDEZ, F. ( 1997 ). Comentarios al Reglamento de los Servicios de Prevención. (Real Decreto 39/1997, de 17 de enero), Tirant lo Blanch, Valencia.

JENNINGS, N.S. (1998). «Visión General de la Minería», en Stellman, J.M. Enciclopedia de salud y seguridad en el trabajo, Ministerio de Trabajo y Asuntos Sociales, Madrid.

Ley $31 / 1995$, de 8 de noviembre, de Prevención de Riesgos Laborales (BOE de 10 de noviembre de 1995).

Ley 54/2003, de 12 de diciembre, de Reforma del Marco Normativo de la Prevención de Riesgos Laborales (BOE de 13 de diciembre de 2003).

MINISTERIO DE TRABAJO y ASUNTOS SOCIALES. (2002). Anuario de Estadísticas Laborales y de Asuntos Sociales 2002. Ministerio de Trabajo y Asuntos Sociales, Madrid.

MINISTERIO DE TRABAJO E INMIGRACION. (2008). Anuario de Estadísticas del Ministerio de Trabajo e inmigración 2007. Ministerio de Trabajo e Inmigración, Madrid.

NIEDERLEYTNER, J.A., GRAU, M. y YANES, J. ( 1996). Gestión de la Prevención en las Empresas: Cómo Gestionar la Prevención. Edición Especial CINCO DÍAS, Madrid.

O'TOOLE, M. (2002). «The Relationship between Employees' Perceptions of Safety and Organizational Culture», Journal of Safety Research 33, pp. 231-243.

OLIVEIRA, J.C. DE y COELHO, D.A. (2002). «The integration of standards systems of quality management, environmental management and occupational health and safety management», International Journal of Production Research 40, pp. 3857-3866. 
ORGANIZACION INTERNACIONAL DEL TRABAJO (2002). La evolución del empleo, el tiempo de trabajo y la formación en la industria minera. Organización Internacional del Trabajo, Ginebra.

PETERSEN, D. (2000). «Safety management: our strengths \& weaknesses», Professional Safety, January, pp. 16-19.

QUEJIDO, E. (2008). «Gestión de la seguridad y salud laboral: OHSAS 18001:2007», Forum Calidad, n I89, pp. 36-38.

REAL DECRETO 39/1997, de 17 de enero, por el que se aprueba el Reglamento de los Servicios de Prevención (BOE de 31 de enero de 1997).

REAL DECRETO 1389/1997, de 5 de septiembre, sobre disposiciones mínimas destinadas a proteger la seguridad y salud de los trabajadores en las actividades mineras (BOE de 7 de octubre de 1997).

REAL DECRETO 604/2006, de 19 de mayo, por el que se modifican el Real Decreto 39/1997, de 17 de enero, por el que se aprueba el Reglamento de los Servicios de Prevención, y el Real Decreto 1627/1997, de 24 de octubre, por el que se establecen las disposiciones mínimas de seguridad y salud en las obras de construcción (BOE de 29 de mayo de 2006).

REAL DECRETO 337/2010, de 19 de marzo, por el que se modifican el Real Decreto 39/1997, de 17 de enero, por el que se aprueba el Reglamento de los Servicios de Prevención; el Real Decreto I 109/2007, de 24 de agos- to, por el que se desarrolla la Ley 32/2006, de 18 de octubre, reguladora de la subcontratación en el sector de la construcción y el Real Decreto 1627/1997, de 24 de octubre, por el que se establecen disposiciones mínimas de seguridad y salud en obras de construcción (BOE de 23 de marzo de 2010).

RIESGO, P.; SUAREZ, A.; IGLESIAS, F.J. (2005). «Hacia un modelo de sistemas de gestión de la seguridad y salud laboral según el estándar OHSAS en el sector de los bienes de equipo», Actas del IX Congreso de Ingeniería de Organización, Gijón.

RUBIO, J.C. (200I). «Sistemas de gestión de la seguridad y salud en el trabajo». Prevención, Trabajo y Salud I4, pp. $4-13$.

SALOMONE, R. (2008). «Integrated management systems: experiences in Italian organizations», Journal of Cleaning Production 16, pp. 1786- 1806.

SESE, A.; PALMER, A.L.; MONTANO, J.J.; JIMENEZ, R. y LLORENS, N. (2002). «Occupational safety and health in Spain», Journal of Safety Research 33, pp. 5 I I-525.

ZENG, S.X.; SHI, J.J.y LOU, G.X. (2007). «A synergetic model for implementing an integrated management system: an empirical study in China», Journal of Cleaner Production I5, pp. 1760- 1767.

ZENG, S.X., TAM, V.W.Y. y TAM, C.M. (2008). «Towards occupational health and safety systems in the construction industry of China». Safety Science 46, pp. I I 55- I 168. 
ANEXO

Similitudes entre OHSAS I800 I:2007, ISO |400 I:2004 e ISO 900 I:2000

\begin{tabular}{|c|c|c|c|c|c|}
\hline \multicolumn{2}{|r|}{ OHSAS I800I:2007 } & \multicolumn{2}{|r|}{ ISO |400I:2004 } & \multicolumn{2}{|r|}{ ISO $9001: 2000$} \\
\hline- & Introducción & - & Introducción & $\begin{array}{l}0 \\
0.1 \\
0.2 \\
0.3 \\
0.4\end{array}$ & $\begin{array}{l}\text { Introducción } \\
\text { Generalidades } \\
\text { Enfoque basado en procesos } \\
\text { Relación con la Norma ISO } 9004 \\
\text { Compatibilidad con otros sistemas de } \\
\text { gestión }\end{array}$ \\
\hline 1 & Objeto y campo de aplicación & 1 & Objeto y campo de aplicación & $\begin{array}{l}\mathrm{I} \\
\mathrm{I} . \mathrm{I} \\
\mathrm{I} .2\end{array}$ & $\begin{array}{l}\text { Objeto y campo de aplicación } \\
\text { Generalidades } \\
\text { Aplicación }\end{array}$ \\
\hline 2 & Publicaciones para consulta & 2 & Normas para consulta & 2 & Normas para consulta \\
\hline 3 & Términos y definiciones & 3 & Términos y definiciones & 3 & Términos y definiciones \\
\hline 4 & $\begin{array}{l}\text { Requisitos del sistema de gestión } \\
\text { de la SST (título solamente) }\end{array}$ & 4 & $\begin{array}{l}\text { Requisitos del sistema de gestión } \\
\text { ambiental (título solamente) }\end{array}$ & 4 & $\begin{array}{l}\text { Sistema de gestión de la calidad } \\
\text { (título solamente) }\end{array}$ \\
\hline 4.1 & Requisitos generales & 4.1 & Requisitos generales & $\begin{array}{l}4.1 \\
5.5 \\
5.5 .1\end{array}$ & $\begin{array}{l}\text { Requisitos generales } \\
\text { Responsabilidad, autoridad y comunica- } \\
\text { ción } \\
\text { Responsabilidad y autoridad }\end{array}$ \\
\hline 4.2 & Política de SST & 4.2 & Política ambiental & $\begin{array}{l}5.1 \\
5.3 \\
8.5\end{array}$ & $\begin{array}{l}\text { Compromiso de la dirección } \\
\text { Política de la calidad } \\
\text { Mejora continua }\end{array}$ \\
\hline 4.3 & Planificación (título solamente) & 4.3 & Planificación (título solamente) & 5.4 & Planificación (título solamente) \\
\hline 4.3 .1 & $\begin{array}{l}\text { Identificación de peligros, } \\
\text { evaluación de riesgos y } \\
\text { determinación de controles }\end{array}$ & 4.3.1 & Aspectos ambientales & $\begin{array}{c}5.2 \\
7.2 .1 \\
7.2 .2\end{array}$ & $\begin{array}{l}\text { Enfoque al cliente } \\
\text { Determinación de los requisitos relacio- } \\
\text { nados con el producto } \\
\text { Revisión de los requisitos } \\
\text { relacionados con el producto }\end{array}$ \\
\hline 4.3 .2 & $\begin{array}{l}\text { Requisitos legales y otros } \\
\text { requisitos }\end{array}$ & 4.3 .2 & $\begin{array}{l}\text { Requisitos legales y otros } \\
\text { requisitos }\end{array}$ & $\begin{array}{c}5.2 \\
7.2 .1\end{array}$ & $\begin{array}{l}\text { Enfoque al cliente } \\
\text { Determinación de los requisitos relacio- } \\
\text { nados con el producto }\end{array}$ \\
\hline 4.3 .3 & Objetivos y programas & 4.3 .3 & Objetivos, metas y programa & $\begin{array}{l}5.4 .1 \\
5.4 .2 \\
8.5 .1\end{array}$ & $\begin{array}{l}\text { Objetivos de la calidad } \\
\text { Planificación del sistema de gestión de la } \\
\text { calidad } \\
\text { Mejora continua }\end{array}$ \\
\hline 4.4 & $\begin{array}{l}\text { Implementación y operación } \\
\text { (título solamente) }\end{array}$ & 4.4 & $\begin{array}{l}\text { Implementación y operación (tí- } \\
\text { tulo solamente) }\end{array}$ & 7 & $\begin{array}{l}\text { Realización del producto (título } \\
\text { solamente) }\end{array}$ \\
\hline 4.4 .1 & $\begin{array}{l}\text { Recursos, funciones, } \\
\text { responsabilidad y autoridad }\end{array}$ & 4.4.I & $\begin{array}{l}\text { Recursos, funciones, } \\
\text { responsabilidad y autoridad }\end{array}$ & $\begin{array}{l}5.1 \\
5.5 .1 \\
5.5 .2 \\
6.1 \\
6.3\end{array}$ & $\begin{array}{l}\text { Compromiso de la dirección } \\
\text { Responsabilidad y autoridad } \\
\text { Representante de la dirección } \\
\text { Provisión de recursos } \\
\text { Infraestructura }\end{array}$ \\
\hline 4.4 .2 & $\begin{array}{l}\text { Competencia, formación y toma } \\
\text { de conciencia }\end{array}$ & 4.4 .2 & $\begin{array}{l}\text { Competencia, formación y toma } \\
\text { de conciencia }\end{array}$ & $\begin{array}{l}6.2 .1 \\
6.2 .2\end{array}$ & $\begin{array}{l}\text { (Recursos humanos) } \\
\text { Generalidades } \\
\text { Competencia, toma de conciencia } \\
\text { y formación }\end{array}$ \\
\hline 4.4 .3 & $\begin{array}{l}\text { Comunicación, participación y } \\
\text { consulta }\end{array}$ & 4.4 .3 & Comunicación & $\begin{array}{l}5.5 .3 \\
7.2 .3\end{array}$ & $\begin{array}{l}\text { Comunicación interna } \\
\text { Comunicación con el cliente }\end{array}$ \\
\hline 4.4 .4 & Documentación & 4.4 .4 & Documentación & 4.2 .1 & $\begin{array}{l}\text { (Requisitos de la documentación) } \\
\text { Generalidades }\end{array}$ \\
\hline 4.4.5 & Control de documentos & 4.4 .5 & Control de documentos & 4.2 .3 & Control de los documentos \\
\hline
\end{tabular}


(continuación)

\begin{tabular}{|c|c|c|c|c|c|}
\hline \multicolumn{2}{|r|}{ OHSAS I800I:2007 } & \multicolumn{2}{|r|}{ ISO | 400I:2004 } & \multicolumn{2}{|r|}{ ISO $9001: 2000$} \\
\hline 4.4 .6 & Control operacional & 4.4 .6 & Control operacional & $\begin{array}{l}7.1 \\
7.2 \\
7.2 .1 \\
7.2 .2 \\
7.3 .1 \\
7.3 .2 \\
7.3 .3 \\
7.3 .4 \\
7.3 .5 \\
7.3 .6 \\
7.3 .7 \\
7.4 .1 \\
7.4 .2 \\
7.4 .3 \\
7.5 \\
7.5 .1 \\
7.5 .2 \\
7.5 .5\end{array}$ & $\begin{array}{l}\text { Planificación de la realización del producto } \\
\text { Procesos relacionados con el cliente } \\
\text { Determinación de los requisitos } \\
\text { relacionados con el producto } \\
\text { Revisión de los requisitos relacionados } \\
\text { con el producto } \\
\text { Planificación del diseño y desarrollo } \\
\text { Elementos de entrada para el diseño y } \\
\text { desarrollo } \\
\text { Resultados del diseño y desarrollo } \\
\text { Revisión del diseño desarrollo } \\
\text { Verificación del diseño y desarrollo } \\
\text { Validación del diseño y desarrollo } \\
\text { Control de los cambios del diseño y } \\
\text { desarrollo } \\
\text { Proceso de compras } \\
\text { Información de las compras } \\
\text { Verificación de los productos comprados } \\
\text { Producción y prestación del servicio } \\
\text { Control de la producción y de la } \\
\text { prestación del servicio } \\
\text { Validación de los procesos de la } \\
\text { producción y de la prestación del servicio } \\
\text { Preservación del producto }\end{array}$ \\
\hline 4.4 .7 & $\begin{array}{l}\text { Preparación y respuesta ante } \\
\text { emergencias }\end{array}$ & 4.4 .7 & $\begin{array}{l}\text { Preparación y respuesta ante } \\
\text { emergencias }\end{array}$ & 8.3 & Control del producto no conforme \\
\hline 4.5 & Verificación (título solamente) & 4.5 & Verificación (título solamente) & 8 & $\begin{array}{l}\text { Medición, análisis y mejora (título } \\
\text { solamente) }\end{array}$ \\
\hline 4.5 .1 & $\begin{array}{l}\text { Seguimiento y medición del } \\
\text { desempeño }\end{array}$ & 4.5 .1 & Seguimiento y medición & $\begin{array}{c}7.6 \\
\\
8.1 \\
8.2 .3 \\
8.2 .4 \\
8.4\end{array}$ & $\begin{array}{l}\text { Control de los dispositivos de } \\
\text { seguimiento y de medición } \\
\text { (Medición, análisis y mejora) } \\
\text { Generalidades } \\
\text { Seguimiento y medición de los procesos } \\
\text { Seguimiento y medición del producto } \\
\text { Análisis de datos }\end{array}$ \\
\hline 4.5 .2 & $\begin{array}{l}\text { Evaluación del cumplimiento } \\
\text { legal }\end{array}$ & 4.5 .2 & $\begin{array}{l}\text { Evaluación del cumplimiento } \\
\text { legal }\end{array}$ & $\begin{array}{l}8.2 .3 \\
8.2 .4\end{array}$ & $\begin{array}{l}\text { Seguimiento y medición de los procesos } \\
\text { Seguimiento y medición del producto }\end{array}$ \\
\hline 4.5 .3 & $\begin{array}{l}\text { Investigación de incidentes, no } \\
\text { conformidad, acción correctiva y } \\
\text { acción preventiva (título solamente) }\end{array}$ & - & - & - & - \\
\hline 4.5.3.1 & Investigación de incidentes & - & - & - & - \\
\hline 4.5.3.2 & $\begin{array}{l}\text { No conformidad, acción correcti- } \\
\text { va y acción preventiva }\end{array}$ & 4.5 .2 & $\begin{array}{l}\text { No conformidad, acción } \\
\text { correctiva y acción preventiva }\end{array}$ & $\begin{array}{c}8.3 \\
8.4 \\
8.5 .2 \\
8.5 .3\end{array}$ & $\begin{array}{l}\text { Control del producto no conforme } \\
\text { Análisis de datos } \\
\text { Acción correctiva } \\
\text { Acción preventiva }\end{array}$ \\
\hline 4.5 .4 & Control de los registros & 4.5 .4 & Control de los registros & 4.2 .4 & Control de los registros \\
\hline 4.5 .5 & Auditoría interna & 4.5 .5 & Auditoría interna & 8.2 .2 & Auditoría interna \\
\hline 4.6 & Revisión por la dirección & 4.6 & Revisión por la dirección & $\begin{array}{l}5.1 \\
5.6 \\
\\
5.6 .1 \\
5.6 .2 \\
5.6 .3 \\
8.5 .1\end{array}$ & $\begin{array}{l}\text { Compromiso de la dirección } \\
\text { Revisión por la dirección (título } \\
\text { solamente) } \\
\text { Generalidades } \\
\text { Información para la revisión } \\
\text { Resultados de la revisión } \\
\text { Mejora continua }\end{array}$ \\
\hline
\end{tabular}

